\title{
Neoplasias en pacientes con infección por VIH. Estudio descriptivo de 129 casos en el período 1993-2010*
}

\author{
Héctor Meijide, Álvaro Mena, Berta Pernas, Ángeles Castro, Soledad López, Pilar Vázquez, \\ Laura Bello, Josefa Baliñas, Guillermo Rodríguez-Martínez y José D Pedreira
}

\footnotetext{
Hospital Universitario de A Coruña. A Coruña, España. Servicio de Medicina Interna Unidad de VIH (HM, AM, BP, AC, SL, $P V, L B, J B, J D P)$ Unidad de Admisión y Documentación Clínica (GRM)

Los autores reconocen no presentar

ningún conflicto de intereses. Financiamiento: Estudio realizado como parte del trabajo habitual.

*Este trabajo ha sido galardonado con el tercer premio de la I Edición de Premios de Investigación de la Sociedad Gallega Interdisciplinaria de SIDA (SOGAISIDA).

Recibido: 4 de julio de 2012 Aceptado: 11 de marzo de 2013

Correspondencia a: Héctor Meijide Míguez hectormeijide@hotmail.com
}

\section{Malignancies in HIV-infected patients. Descriptive study of 129 cases between 1993 and 2010*}

Introduction: The development of malignancies is a problem associated with HIV infection. The incidence and spectrum of malignancies has been modified with the addition of highly active antiretroviral therapy (HAART). Aim: To describe the clinical and epidemiological characteristics and prognosis of HIV patients who have developed a malignancy. Methods: Retrospective observational study was conducted in HIV + patients who developed a malignancy between 1993-2010 in a referral hospital. AIDS-defining malignancies (ADN) and non-AIDS-defining malignancies (NADN) were compared. Results: 125 patients were identified with at least one malignancy. The most frequent malignancies were: non-Hodgkin lymphoma (n; 39; 30.2\%), Kaposi's sarcoma (n: 20; 15.5\%), Hodgkin's disease (n: 11;8.8\%), lung cancer (n: 20;15.5\%) and hepatocellular carcinoma (n: $9 ; 6.9 \%)$. The mean age was $42 \pm 11$ years, $84 \%$ male, $55.8 \%$ were coinfected with HBV and or HCV. The risk behaviors were: $45.6 \%$ intravenous drug users, $16.8 \%$ men who have sex with men and 20\% heterosexuals). There were 67 (52\%) NADN and $62(48 \%)$ ADN; NADN patients had a longer story of HIV infection and longer exposure to HAART, better level of immunodeficiency and better virological control than ADN patients. Four patients developed a second malignancy. Overall survival was $34.7 \%$. Conclusions: We found an increased incidence of NADN, appearing in patients with better virological and immunological control than ADN group. Mortality of patients with HIV infection and malignancy is still very high.

Key words: HIV, malignancy, cancer, AIDS.

Palabras clave: VIH, tumor, cáncer, SIDA.

\section{Introducción}

$\mathrm{D}$ esde los inicios de la epidemia de síndrome de inmunodeficiencia adquirida (SIDA) a principios de la década de los 80 , el cáncer ha figurado en un lugar destacado como una enfermedad oportunista. Los dos máximos exponentes de neoplasias asociadas a SIDA son el sarcoma de Kaposi (SK) y el linfoma no Hodgkin (LNH), ambos incrementados paralelamente con la progresión de inmunodeficiencia celular. El cáncer de cérvix $(\mathrm{CC})$, aunque menos común que los anteriores, también está considerado como tumor definitorio de SIDA(NDS) $)^{1-5}$.

Con la instauración del tratamiento anti-retroviral de gran actividad (TARGA) en 1996, la morbi-mortalidad inherente a la infección por el virus de la inmunodeficiencia humana (VIH) ha disminuido ${ }^{6-8}$, especialmente aquella relacionada con procesos infecciosos oportunistas. $\mathrm{Al}$ igual que otras enfermedades oportunistas, las neoplasias asociadas a SIDA (SK y LNH) también han bajado su incidencia desde 1996; no obstante la tasa de CC no se ha visto modificada ${ }^{9-13}$. Además se ha objetivado un incre- mento importante de procesos tumorales no definitorios de SIDA (NNDS) respecto a los NDS, contrastado en estudios previos en comparación con el resto de población general ${ }^{14}$. Numerosas hipótesis han salido a la luz para intentar explicar este fenómeno. Por un lado, se sabe que la propia infección por el VIH condiciona defectos en la inmunidad celular, con la subsiguiente alteración en los sistemas de vigilancia y supresión de aquellos virus con potencial oncogénico ${ }^{15}$, teniendo en cuenta que muchos de estos pacientes están co-infectados por otros virus como el papiloma humano (VPH), Epstein Barr (VEB), hepatitis $\mathrm{B}$ (VHB) y hepatitis C (VHC). Interesantes estudios han mostrado la asociación de determinados virus con cáncer, como el caso del LH con el VEB ${ }^{16,17}$, el hepatocarcinoma con el VHB y el VHC ${ }^{18,19}$ o el cáncer de ano /pene /vulva /cavidad oral con el $\mathrm{VPH}^{20-29}$. Por otro lado, se cree que el propio tratamiento anti-retroviral conlleva una activación crónica del sistema inmune, y por tanto, podría contribuir a incrementar el riesgo de algunos tumores. El aumento de la esperanza de vida de la población infectada con $\mathrm{VIH}$, asociado al mayor envejecimiento de la misma, puede jugar un papel importante en el mayor desarrollo de 
tumores y de segundas neoplasias en este colectivo ${ }^{30}$. De hecho, muestra del cambio de espectro en esta población es que el número actual de individuos con infección VIH mayores de 50 años se ha multiplicado por cinco con relación a antes de $1996^{31}$. Finalmente, algunos factores de riesgo clásicos, como el alcohol o el tabaco, son más prevalentes en población infectada por VIH que en la población general ${ }^{32}$.

\section{Objetivos}

Describir las características clínicas y epidemiológicas de los pacientes infectados por VIH que desarrollaron un cáncer, analizar las diferencias entre NDS y NNDS, la aparición de segundas neoplasias así como el pronóstico de esta cohorte de pacientes.

\section{Material y Métodos}

Se realizó un estudio observacional, retrospectivo, de una cohorte de pacientes con infección por VIH que desarrollaron un cáncer en el periodo comprendido entre 1993 y 2010, seguidos en la Unidad de VIH de un hospital de referencia.

El método de selección se basó en la revisión de las historias clínicas de aquellos pacientes reconocidos por la Unidad de Codificación del centro con los diagnósticos previamente reseñados de infección por VIH y cáncer. Se recogieron datos epidemiológicos (edad, sexo, infección por $\mathrm{VHB} / \mathrm{VHC}$, fecha de su última revisión y fecha de muerte, según procediese), datos relacionados con la infección por VIH (fecha de diagnóstico de la infección, grupo de riesgo, carga viral, recuento de CD4, tratamiento anti-retroviral y existencia de SIDA previo) y datos relacionados con la neoplasia (fecha de diagnóstico, tipo de tumor y tratamiento).

Los tumores se clasificaron en dos grupos: NDS y NNDS. No se consideró para el presente estudio el análisis de las neoplasias cervicales intra-epiteliales (CIN). Se definió como segundo cáncer la aparición, simultánea o no, de otro tumor distinto, con histología maligna, en el mismo o diferente órgano que el primero, y se excluyó la posibilidad de que uno fuese metástasis de otro.

\section{Análisis estadístico}

Las variables cuantitativas se expresaron como media y desviación estándar (salvo que se indique otra manera) y las cualitativas como frecuencias y porcentajes. Se compararon las variables descritas entre los grupos NDS y NNDS, las cualitativas mediante $\chi^{2}$ o test exacto de Fisher y las cuantitativas mediante t-Student o el test de Mann-Whitney, según correspondiese. El análisis de supervivencia se realizó mediante las curvas de KaplanMeier. En todos los casos se consideró que los resultados fueron estadísticamente significativos cuando $\mathrm{p}<0,05$. El procesamiento y análisis estadístico de los datos recogidos se realizó mediante el paquete estadístico SPSS 18.0.

\section{Resultados}

Se identificaron 129 neoplasias en 125 pacientes. El tumor más frecuentemente encontrado fue el LNH (39 pacientes), seguido por el SK (20 pacientes) y la neoplasia de pulmón (20 pacientes). La edad media al diagnóstico del tumor fue de $42 \pm 11$ años, la mayoría fueron varones (84\%) y en $55,8 \%$ se constató co-infección por $\mathrm{VHC}$ y/o VHB. Respecto a las conductas de riesgo: $45,6 \%$ eran usuarios de drogas vía parenteral (UDVP), 16,8\% hombres con relaciones sexuales con hombres (HSH), $20 \%$ heterosexuales y $17,6 \%$ no reconoció práctica de riesgo alguna. El 59\% tenía una carga viral detectable al diagnóstico. El recuento de CD4 fue inferior a 200 céls/ $\mathrm{mm}^{3}$ en $41 \%$ de los casos y $51 \%$ tomaba tratamiento anti-retroviral previo al diagnóstico del tumor.

\section{Tipo de neoplasia}

Respecto al tipo de neoplasia, se encontraron 16 tipos de tumor según la histología y la localización. (Figura 1). El más frecuente fue el LNH con 39 casos $(30,2 \%)$; de ellos, 9 fueron linfoma tipo Burkitt. El 15,5\% presentó SK (20 casos). Completa el apartado de NDS el CC con 3 casos (2,3\%). De los 59 pacientes que presentaron un primer NDS, en 21 (36\%) se diagnosticó la infección por VIH de manera simultánea a la NDS y en 39 de ellos $(66 \%)$ fue la primera manifestación de SIDA.

En cuanto a las NNDS, la neoplasia sólida de pulmón fue el tumor más frecuente, con 20 casos (15,5\%), seguido por el linfoma de Hodgkin (LH) con 11 casos (8,5\%), hepatocarcinoma con 9 casos $(6,9 \%)$ y $\mathrm{Ca}$ anal con 4 casos $(3,1 \%)$. Completan el listado de tumores 23 casos (17,8\%): Ca del área otorrinolaringológica (ORL) (n: 6),

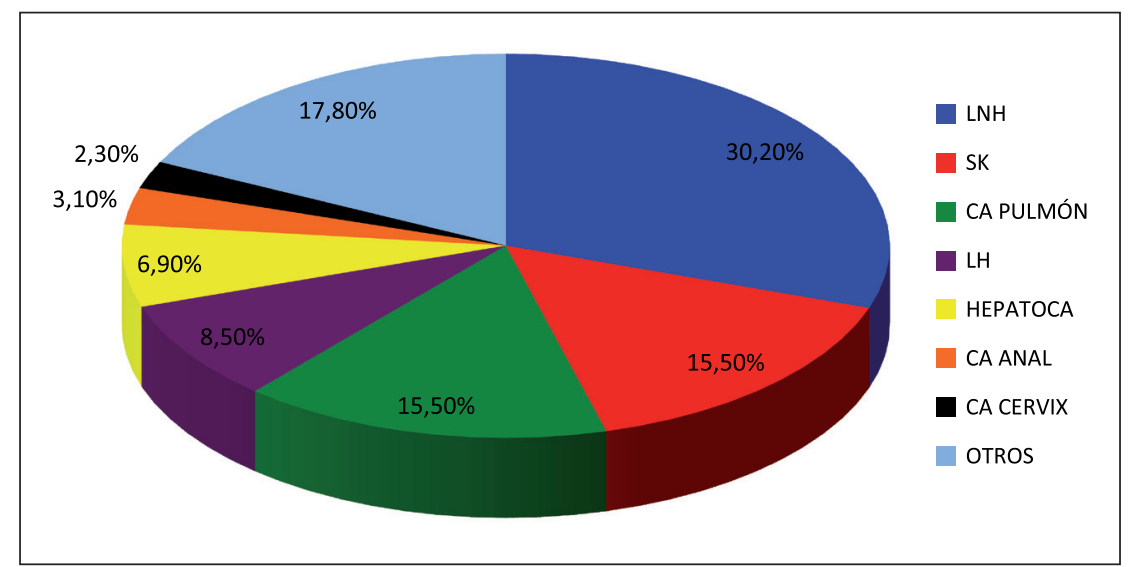

Figura 1. Tipo de neoplasia. 
Ca de pene (n: 3), Ca de origen digestivo (n: 3), germinoma (n: 3), Ca de próstata (n: 2), leucemia (n: 1), Ca de mama (n: 1), Ca de vejiga (n: 1) y por último Ca primario desconocido (n: 3). En 5 casos (7,6\%) se diagnosticó de manera simultánea la NNDS y la infección por VIH.

\section{Neoplasias definitorias de SIDA y neoplasias no definitorias de SIDA}

En esta cohorte de 125 pacientes, se encuentran 66 NNDS (53\%) y 59 NDS (47\%), como primera neoplasia. (Tabla 1).

El porcentaje de UDVP fue mayor en el grupo de NNDS, mientras que el de HSH lo fue en el de NDS, diferencias estadísticamente significativas (Tabla 1). Por otro lado, el grupo de NNDS está formado por pacientes con mayor tiempo de evolución de la infección por $\mathrm{VIH}$, que con más frecuencia reciben TARGA y durante mayor tiempo, con mejor situación inmunológica y mejor control virológico que los del grupo de NDS.

En el periodo estudiado se observó una variación en la distribución, $65 \%$ de las neoplasias diagnosticadas antes del año 2000 se catalogaron como NDS y 35\% NNDS mientras que posteriormente a esta fecha, $42 \%$ fueron NDS y 58\% NNDS (p: 0,03).

No se encontró diferencias significativas entre ambos grupos (p: 0,12) al analizar las curvas de supervivencia de Kaplan-Meier (Figura 2). La supervivencia global fue de $39,7 \%$, con un seguimiento de hasta 17 años.

\begin{tabular}{|c|c|c|c|}
\hline & $\begin{array}{l}\text { NDS } \\
\text { (n: 59) }\end{array}$ & $\begin{array}{l}\text { NNDS } \\
\text { (n: 66) }\end{array}$ & $\mathbf{P}$ \\
\hline Edad (años) & $38(32-44)$ & $43(37-49)$ & 0,005 \\
\hline Sexo varón & $51(86)$ & $54(82)$ & ns \\
\hline \multicolumn{4}{|l|}{ Grupo de riesgo: } \\
\hline UDVP & $21(45)$ & $36(64)$ & 0,036 \\
\hline $\mathrm{HSH}$ & $14(30)$ & $7(12)$ & 0,027 \\
\hline Heterosexual & $12(25)$ & $13(24)$ & ns \\
\hline Tiempo de infección por VIH (años) & $1(0-9)$ & $8(3-14)$ & $<0,001$ \\
\hline TARGA previo & $18(30)$ & $46(70)$ & $<0,001$ \\
\hline Tiempo de TARGA (meses) & $36(12-51)$ & $72(40-120)$ & 0,003 \\
\hline Categoría C previa & $20(34)$ & $22(33)$ & ns \\
\hline CD4 < 200céls $/ \mathrm{mm}^{3}$ & $31(53)$ & $20(30)$ & 0,018 \\
\hline CV indetectable & $13(22)$ & $38(58)$ & $<0,001$ \\
\hline Co-infección VHB y/o VHC & $25(50)$ & $38(60)$ & ns \\
\hline \multicolumn{4}{|c|}{$\begin{array}{l}\text { Variables cualitativas expresadas como n (\%), cuantitativas como mediana (rango intercuartíl). TAR- } \\
\text { GA: tratamiento anti.retroviral de gran actividad. NDS: neoplasia definitoria de SIDA; NNDS: neoplasia } \\
\text { no definitoria de SIDA; UDVP: usuarios de drogas vía parenteral; HSH: hombres con relaciones sexuales } \\
\text { con hombres; CV: carga viral del VIH; VHB: virus de la hepatitis B; VHC: virus de la hepatitis C; ns: no } \\
\text { significativo }(p>0,05) \text {. }\end{array}$} \\
\hline
\end{tabular}

\section{Segundas neoplasias}

Un total de cuatro pacientes $(3,2 \%)$ desarrollaron una segunda neoplasia: dos pacientes con LNH desarrollaron SK, un paciente con SK desarrolló LNH y un paciente con Ca de próstata desarrolló un $\mathrm{Ca}$. epidermoide de pulmón.

\section{Discusión}

En el último informe de infección por VIH/SIDA publicado en 2010, se recogen 1.242 nuevos casos de infección por VIH en Galicia (España) entre 2004 y $2009^{33}$. Al permanecer estable el número de nuevos diagnósticos y transformarse la infección por VIH en una enfermedad crónica, debido, en parte, a la instauración del TARGA, aparecen nuevas co-morbilidades que hasta hace años no se tenían en cuenta en este grupo de pacientes, entre ellas los cánceres, la enfermedad cardiovascular y las enfermedades respiratorias crónicas, en detrimento de las complicaciones fundamentalmente de tipo infeccioso que presentaban los pacientes con VIH en épocas previas.

La incidencia de NNDS en la cohorte de EuroSIDA ${ }^{30}$ entre 1994 y 2007 fue de 4,3 por 1.000 personas-año en el seguimiento. Se observó que un mayor recuento de linfocitos T CD4 se asociaba de manera independiente a una disminución de la incidencia de neoplasias, no sólo de los NDS sino también de las NNDS. Numerosas infecciones virales han sido relacionadas con la aparición de neoplasias; se estima que cerca de $15 \%$ de todos los cánceres

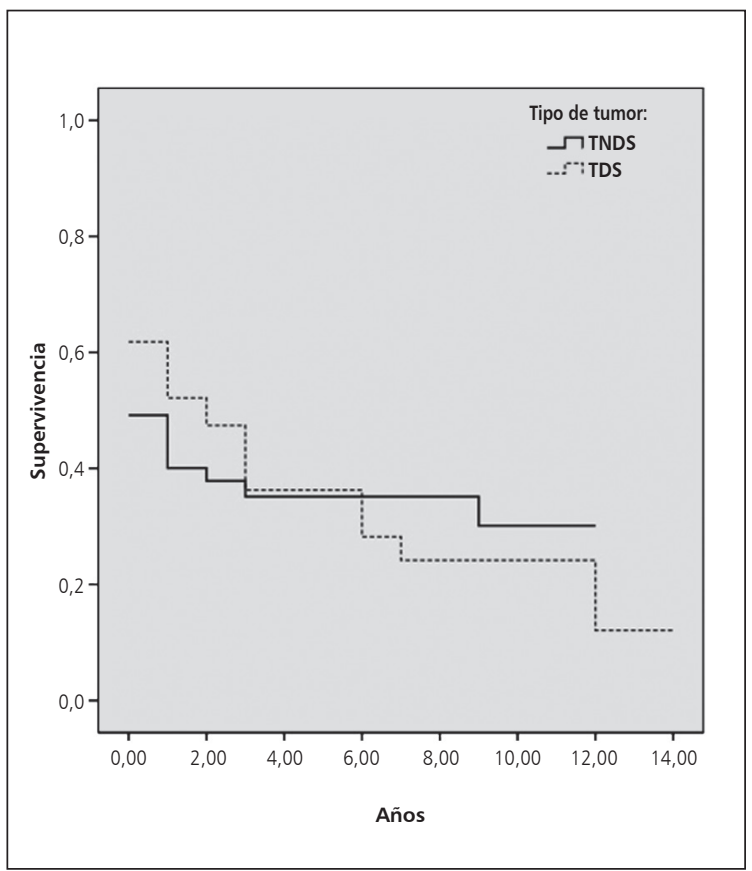

Figura 2. Curvas de supervivencia de Kaplan-Meier de los tumores definitorios de SIDA (TDS) y los tumores no definitorios de SIDA (TNDS). $\mathrm{P}=0,12$. 
tiene un agente infeccioso en su origen ${ }^{20}$. Paralelamente también se ha constatado que muchas de las neoplasias que aparecen en pacientes infectados por el VIH están directamente relacionadas con infecciones virales, aunque el VIH no sea en sí mismo un virus oncogénico ${ }^{34}$. Prueba de ello son conocidas las relaciones entre VPH con el cáncer de cérvix, ano, pene, vulva u orofaringe; el VHB y VHC con el hepatocarcinoma, y el VEB con el LH, entre otros. Estos virus son mucho más prevalentes en población infectada por VIH que no infectada y con frecuencia son más virulentos y con mayor potencial oncogénico en coinfectados. Así pues, en pacientes con infección por VIH y co-infección por virus hepatotropos, la fibrosis progresa de manera más rápida y la incidencia de hepatocarcinoma es mayor; de manera paralela, ocurre algo similar con la infección por VPH o VEB.

En el presente estudio se describen las características de 125 pacientes infectados por el VIH que desarrollaron algún tumor durante un periodo de 17 años de seguimiento. A pesar de las limitaciones inherentes al carácter retrospectivo del mismo, los resultados no difieren en gran medida de otras series descritas previamente. Aunque los tumores más frecuentemente encontrados son el LNH y el SK, ambos NDS, en el cómputo global, más de la mitad de pacientes tuvo un NNDS, lo cual refleja la tendencia actual al aumento de este tipo de tumores en detrimento de los NDS, tal y como han reflejado estudios previos ${ }^{35-39}$, desde la introducción del tratamiento anti-retroviral, disminuyendo la incidencia hasta cinco veces respecto a la era pre-TARGA. De manera global, en nuestra serie hemos documentado poco más de 3 tumores/año antes del año 2000 con un predominio claro de los NDS (65 vs $35 \%$ ) y casi 9 tumores/año después de este año con más NNDS (58\%) que NDS (42\%), datos que son muy significativos. Respecto al CC tan sólo hemos encontrado tres casos en nuestra serie, si bien una limitación es el no haber tenido en cuenta las lesiones cervicales intraepiteliales, que como bien es conocido, tienen un riesgo mucho mayor de desarrollar $\mathrm{Ca}$ invasor, especialmente en mujeres infectadas por el VIH.

Analizando los NNDS, en nuestra serie destacamos un elevado número de neoplasias sólidas de pulmón, muy por encima de los números que presentan otras series, como la de Ramírez-Olivencia y cols. ${ }^{40}$. Un factor a tener en cuenta es que nuestro hospital es centro de referencia de cirugía torácica, lo que puede ser un sesgo de selección. Obviamente, la mejora en la supervivencia de los pacientes VIH, el incremento en la edad de los pacientes infectados y la persistencia del hábito tabáquico harán que la incidencia de estos tumores pueda ir en aumento. En nuestra serie no se recogió el hábito tabáquico, si bien se ha constatado en algunos trabajos el incremento de neoplasias de pulmón en pacientes con infección por VIH, independientemente de la edad y del hábito tabáquico, propugnando como hipótesis la propia inmunosupresión celular mantenida y los efectos sobre la inmunidad del tratamiento anti-retroviral ${ }^{41-42}$.

Dentro de las NNDS cabe destacar por su frecuencia e importancia el hepatocarcinoma, que alcanzó en nuestra serie a 7,2\% de los casos. De ellos, 100\% estaban co-infectados por VHC y/o VHB. Por el carácter retrospectivo del estudio, no se pudieron obtener variables importantes como el consumo de alcohol o la presencia previa de cirrosis. La mejor manera de prevenir la aparición de hepatocarcinoma es el tratamiento de las hepatitis víricas, así como la implantación de programas de cribado. La mortalidad inherente a este tipo de cáncer todavía es muy elevada, en nuestra serie fallecen cinco de nueve pacientes durante el seguimiento, resultados similares a los de otras series ${ }^{43-44}$.

También es importante destacar los cánceres relacionados con el VPH, además del CC ya mencionado. Si los englobamos, resultan en un número total de 13 casos (cuatro cánceres de ano, tres cánceres de pene y seis cánceres ORL). En el análisis por subgrupos, se constató que entre los pacientes con neoplasias asociadas a VPH, la mayoría correspondió a $\mathrm{HSH}$, con buena adherencia al TARGA y buen control virológico e inmunológico de la infección por VIH. La infección por VPH en población infectada por VIH es mucho más frecuente que en población general; así, en mujeres con infección por VIH la prevalencia de co-infección cervical por VPH se multiplica por tres respecto a las sin infección por VIH. Este aumento en la prevalencia es mucho más acusado en varones, especialmente en HSH seropositivos para $\mathrm{VIH}$, donde la prevalencia de infección anal por VPH asciende a $89 \%$ en algunas series ${ }^{45}$. Además de una mayor prevalencia, los pacientes infectados por VIH tardan más en aclarar la co-infección por VPH lo que se relaciona al mayor número de procesos neoplásicos asociados a este virus en población seropositiva para VIH. Por ello, se están implantando distintos programas para el diagnóstico precoz de estos tumores asociados al VPH y de las lesiones pre-cancerosas, en base a la experiencia positiva del cribado de displasia de cérvix.

Al igual que otras series ${ }^{33-36}$, los pacientes con NDS tenían una carga viral más elevada y un número de linfocitos T CD4 (en el momento del diagnostico del cáncer) menor que en el grupo de pacientes con NNDS. Es significativo que en $36 \%$ de los NDS, la neoplasia ha llevado al diagnóstico de la infección por VIH, lo que traduce cierto retraso en el diagnóstico de la infección, probablemente de carácter multifactorial. Por otro lado, y aunque en nuestra serie la incidencia de segundos tumores fue baja (cuatro pacientes), sí objetivamos que la mayoría de ellos eran relacionados con infecciones añadidas al VIH (VHS-8, VEB), en probable relación con la mayor inmunosupresión que confiere el tratamiento oncológico 
agresivo del primer cáncer a la ya pre-establecida por el propio VIH. El recuento bajo de CD4 se ha asociado claramente a un mayor riesgo de desarrollo de NDS, sin estar clara la asociación con los NNDS, con estudios que sí la encuentran ${ }^{46}$ y otros que no ${ }^{47}$. Parece que en el desarrollo de NNDS el valor pronóstico del nadir de CD4 es mucho mayor que la cifra de CD4 al diagnóstico del tumor ${ }^{48}$. Además, con los resultados del estudio SMART ${ }^{49}$, se sabe que la suspensión del TARGA conlleva un mayor riesgo de desarrollar NDS. Hay discrepancias en la relación entre la exposición al TARGA y el desarrollo de $\mathrm{NNDS}^{47,50}$, probablemente en relación con los distintos enfoques y análisis; pero todas las guías de manejo de pacientes infectados por VIH son claras en este aspecto, recomendándose el inicio del TARGA cada vez más precoz.

Nuestro estudio tiene limitaciones, en relación con su naturaleza observacional, retrospectiva, con los sesgos de información y de selección que esto conlleva; así como la pérdida de datos en relación con los antecedentes de tabaquismo, alcohol y otros carcinogénicos que podrían tener implicaciones en los resultados. Dadas las condiciones del estudio sólo se ha reflejado la supervivencia global al final del mismo. Existe una tendencia de mayor supervivencia en las NNDS que en las NDS, aunque no se alcanzó la significación estadística.

En conclusión, presentamos una serie amplia de pacientes con infección por VIH y enfermedad tumoral en la que se constata nuevamente el preocupante aumento en la incidencia de neoplasias, sobre todo NNDS, y en especial de neoplasias asociadas a otras infecciones víricas. El diagnóstico precoz de la infección por $\mathrm{VIH}$, el inicio temprano del TARGA eficaz, la modificación de los hábitos de vida y las medidas de cribado de las NDS y NNDS más frecuentes son, posiblemente, las medidas más eficaces para la prevención del desarrollo de estos procesos, aunque se necesitan más estudios que evalúen su impacto.

\section{Resumen}

Introducción: Uno de los problemas asociados a la infección por VIH es el desarrollo de neoplasias. La incidencia y espectro de los distintos cánceres se ha visto modificada con la incorporación del tratamiento anti-retroviral de gran actividad (TARGA). El objetivo del presente estudio es describir las características clínicas y epidemiológicas y el pronóstico de pacientes infectados con VIH que han desarrollado una neoplasia. Material y Métodos: Estudio observacional retrospectivo de una cohorte de pacientes con infección por VIH que desarrollaron algún cáncer en el periodo comprendido entre 1993-2010 en un hospital de referencia. Se compararon las variables entre los casos de neoplasias definitorias de SIDA (NDS) y no definitorios de SIDA (NNDS). Resultados: Se identificaron 125 pacientes con al menos una neoplasia. Los cánceres más frecuentes fueron: linfoma no Hodgkin (n: 39; 30,2\%), sarcoma de Kaposi (n: 20; 15,5\%), enfermedad de Hodgkin (n: 11; 8,8\%), neoplasia pulmón (n: 20; 16\%) y hepatocarcinoma (n: 9; $6,9 \%$ ). La edad media fue $42 \pm 11$ años, $84 \%$ varones, $55,8 \%$ estaban co-infectados por VHB y/o VHC. Las conductas de riesgo fueron: $45,6 \%$ usuarios de drogas vía parenteral, $16,8 \%$ hombres con relaciones sexuales con hombres y $20 \%$ heterosexuales. Se encontraron 67 NNDS (52\%) y $62(48 \%)$ NDS; los pacientes con NNDS presentaron mayor tiempo de evolución de la infección por VIH y de exposición a TARGA, mayor recuento de CD4 y mejor control virológico que los del grupo de NDS. Desarrollaron un segundo tumor cuatro pacientes. La supervivencia global fue de 34,7\%. Conclusiones: Se constata un aumento en la incidencia de NNDS, que se presentan en pacientes con mejor control virológico e inmunológico que los NDS. La mortalidad de los pacientes con infección por VIH y enfermedad tumoral continúa siendo muy elevada.

\section{Referencias bibliográficas}

1.- Anonimous. 1993 revised classification system for HIV infection and expanded surveillance case definition for AIDS among adolescents and adults. MMWR Recomm Rep 1992; 41: 119.

2.- $\quad$ Long J L, Engels E A, Moore R D, Gebo K A. Incidence and outcomes of malignancy in the HAART era in an urban cohort of HIV-infected individuals. AIDS 2008; 22: 489-96.

3.- Engels E A, Pfeiffer R M, Goedert J J, Virgo P, McNeel T, Scoppa S M, et al. Trends in cancer risk among people with AIDS in the United States 1980-2002. AIDS 2006; 20: 1645-54.

4.- Harvard Report on Cancer Prevention. Volume 1: Causes of human cancer. Cancer Causes Control 1996; 7 (suppl 1): S3-59.
5.- Nutankalva L, Wutoh A K, McNeil J, Frederick W R, Reddy R B, Daftary M, et al. Malignancies in HIV: pre- and post-highly active antiretroviral therapy. J Natl Med Assoc 2008; 100: 817-20.

6.- Nuñez M. Hepatotoxicity of antiretrovirals: Incidence, mechanisms and management. J Hepatol 2006; 44: S132-9.

7.- Samaras K. Metabolic consequences and therapeutic options in highly active antiretroviral therapy in human immunodeficiency virus-1 infection. J Antimicrob Chemother 2008; 61: 338-45.

8.- Barbaro G, Barbarini G. HIV infection and cancer in the era of highly active antiretroviral therapy (review). Oncol Rep 2007; 17: 1121-6.

9.- Davis A T, Chakraboryty H, Flowers K,
Mosunjac M V. Cervical dysplasia in women infected with the human immunodeficiency virus (HIV): A correlation with HIV viral load and CD4 count. Gynecol Oncol 2001; 80: 350-4.

10.- Ellerbrock T V, Chiasson M A, Bush T J, Sun X W, Sawo D, Brudney K, et al. Incidence of cervical squamous intraepithelial lesions in HIV-infected woman. JAMA 2000; 283: 1031-7.

11.- Lomalisa P, Smith T, Guidozzi F. Human immunodeficiency virus infection and invasive cervical cancer in South Africa. Gynecol Oncol 2000; 77: 460-3.

12.- Gichangi P B, Bwayo J, Estambale B, De Vuyst H, Ojwang S, Rogo K, et al. Impact of HIV infection on invasive cervical cancer in Kenyan women. AIDS 2003; 17: 1963-8.

13.- Leitao Jr M M, White P, Cracchiolo B. Cervical 
cancer in patients infected with the human immunodeficiency virus. Cancer 2008; 112: 2683-9.

14.- Gates A E, Kaplan L D. AIDS malignancies in the era of highly active antiretroviral therapy. Oncology 2002; 16: 657-65.

15.- Spano J P, Atlan D, Breau J L, Farge D. AIDS and non- AIDS-related malignancies: a new vexing challenge in HIV positive patients. Pt I: Kaposi's sarcoma, non-Hodgkin's lymphoma, and Hodgkin's lymphoma. Eur J Intern Med 2002; 13: 170-9.

16.- Ambinder R F. Epstein-Barr virus associated lymphoproliferations in the AIDS setting. Eur J Cancer 2001; 37: 1209-16.

17.- Glaser S L, Lin R J, Stewart S L, Ambinder R F, Jarrett R F, Brousset P, et al. Epstein-Barr virus associated Hodgkin's disease: epidemiologic characteristics in international data. Int J Cancer 1997; 70: 375-82.

18.- Clifford G M, Rickenbach M, Polesel J. Influence of HIV-related immunodeficiency on the risk of hepatocellular carcinoma. AIDS 2008; 22: 2135-41.

19.- Giordano T P, Kramer J R, Souchek J, Richardson P, El-Serag HB. Cirrhosis and hepatocellular carcinoma in HIV infected veterans with and without the hepatitis $C$ virus: a cohort study, 1992-2001. Arch Intern Med 2004; 164: 2349-54.

20.- Spano J P, Atlan D, Breau J L, Farge D. AIDS and non- AIDS-related malignancies: a new vexing challenge in HIV positive patients. Pt II: Cervical and anal squamous epithelial lesions, lung cancer, testicular germ cell cancers, and skin cancers. Eur J Intern Med 2002; 13: 227-32.

21.- Grulich A E, van Leeuwen M T, Falster M O, Vajdic C M. Incidence of cancers in people with HIV/AIDS compared with immunosuppressed transplant recipients: a meta-analysis. Lancet 2007; 370: 59-67.

22.- Bouvard V, Baan R, Straif K, Grosse Y, Secretan B, El Ghissassi F, et al. A review of human carcinogens-pt B: biological agents. Lancet Oncol 2009; 10: 321-2.

23.- Parkin D M, Bray F. Chapter 2: The burden of HPV-related cancers. Vaccine 2006; 24 (Suppl 3): 11-25.

24.- de Sanjosè S, Palefsky J. Cervical and anal HPV infection in HIV positive women and men. Virus Res 2002; 89: 210-1.

25.- Minkoff H, Ahdieh L, Massad LS, Anastos K, Watts DH, Melnick S, et al. The effect of highly active antiretroviral therapy on cervical cytologic changes AIDS 2001; 15: 2157-64.

26.- Heard I, Tassie J M, Kazatchkine M D, Orth G. Highly active antiretroviral therapy enhances regression of cervical intraepithelial neoplasia in HIV-seropositive women. AIDS 2002; 16: 1799802.

27.- Lillo F B, Ferrari D, Veglia F, Origoni M,
Grasso M A, Lodini S, Mastrorilli E, et al. Human papillomavirus infection and associated cervical disease in human immunodeficiency virus-infected women: effect of highly active antiretroviral therapy. J Infect Dis 2001; 184: 547-51.

28.- Palefsky J. Anal squamous intraepithelial lesions: relation to HIV and human papillomavirus infection. J Acquir Immune Defic Syndr 1999; 21 (suppl 1): S42-8.

29.- Palefsky J M, Holly E A, Ralston M L, Da Costa M, Greenblatt RM: Prevalence and risk factors for anal papillomavirus infection in human immunodeficiency virus (HIV)-positive and high-risk HIV-negative women. J Infect Dis 2001; 183: 383-91.

30.- Mocroft A, Ledergerber B, Katlama C, Kirk O, Reiss P, d’Arminio Monforte A, et al. Decline in the AIDS and death rates in the EuroSIDA study: an observational study. Lancet 2003; 362: 22-9.

31.- Tumbarello M, Rabagliati R, De Gaetano Donati K, Bertagnolio S, Tamburrini E, Tacconelli E et al. Older HIV-positive patients in the era of highly active antiretroviral therapy: changing of a scenario. AIDS 2003;17: 128-31.

32.- Jerico C, Knobel H, Sorli ML, Montero M, Guelar A, Pedro-Botet J. Prevalence of cardiovascular risk factors in $\mathrm{HIV}$-infected patients. Rev Clin Esp 2006; 206: 556-9.

33.- Informe VIH-SIDA en Galicia. Diagnósticos de infección polo VIH (2004-2008); Casos de SIDA (1984-2008). Consellería de Sanidade, Dirección Xeral de Saúde Pública e Planificación. 2009. Xunta de Galicia. Santiago de Compostela.

34.- Pradier C, Carrieri M, Piche M, Rosenthal E, Dellamonica P, Serraino D. HCV infection and cancer risk among HIV-infected individuals. Infection 2003; 3: 194-6.

35.- Berdimo R, Chen R Y, Accortt N A, Raper J L, Linn C, Alison J J, et al. Trends in AIDSdefining and non-AIDS defining malignancies among HIV-infected patients: 1989-2002. Clin Infect Dis 2004; 39: 1380-4.

36.- Bonnet F, Lewden C, May T, Heripret L, Jougla E, Bevilacqua S, et al. Malignancyrelated causes of death in human inmunodeficiency virus-infected patients in the era of highly active antiretroviral therapy. Cancer 2004; 101: 317-24.

37.- Crum N F, Riffenburbh R H, Wegner S, Agan B K, Tasker S A, Spooner K M, et al. Comparisons of causes of death and mortality rates among HIV-infected persons: Analysis of teh pre-, early, and late HAART eras. J Acquir Immune Defic Syndr 2006; 41: 194-200.

38.- Frish M, Biggar R J, Engels E A, Goedert, AIDS-Cancer Match Registry Study Group. Associaton of cancer with AIDS- related immunosuppresion in adults. JAMA 2001; 285 : 1736-45.
39.- Galceran J, Marcos-Gragera R, Soler M, Romaguera A, Ameijide A, Izquierdo A, et al. Cancer incidence in AIDS patients in Catalonia, Spain. Eur J Cancer 2007; 43: 1085-61.

40.- Ramirez-Olivencia G, Valencia-Ortega ME, Martin-Carbonero L, Moreno-Celda V, Gonzalez-Lahoz J. Tumores en pacientes con infección por el virus de la inmunodeficiencia humana: estudio de 139 casos. Med Clin (Bar) 2009; 133: 729-35.

41.- Tirelli U S M, Sandri S, Serraino D, Gobitti C, Fasan M, Sinicco A, et al. Lung carcinoma in 36 patients with human immunodeficiency virus infection. The Italian Cooperative Group on AIDS and Tumors. Cancer 2000, 88: 563-9.

42.- Bower M, Powles T, Nelson M, Shah P, Cox S, Mandelia S, et al. HIV-related lung cancer in the era of HAART. AIDS 2003; 17: 371-75.

43.- Brau N, Fox RK, Xiao P, Marks K, Nakvi Z, Taylor L E, et al. Presentation and outcome of hepatocellular carcinoma in HIV infected patients: A US-Canadian multicenter study. J Hepatol 2007; 47: 527-37.

44.- Giordano T P, Kramer J R, Souchek J, Richardson P, El-Serag H B. Cirrhosis and hepatocellular carcinoma in HIV infected veterans with and without the hepatitis $C$ virus: A cohort study, 1992-2001. Arch Intern Med 2004; 164: 2349-54.

45.- Parisi S G, Cruciani M, Scaggiante R, Boldrin C, Andreis S, Dal Bello F, et al. Anal and oral human papillomavirus infection in HIV-infected subjects in northern Italy: a longitudinal cohort study among men who have sex with men. BMC Infect Dis 2011; 11: 150

46.- Crum-Cianflone N, Hullsiek KH, Marconi V, Weintrob A, Ganesan A, Barthel RV, et al. Trends in the incidence of cancers among HIVinfected persons and the impact of antiretrovira therapy: a 20-years cohort study. AIDS 2009; 23: 41-50.

47.- Baker J V, Peng G, Rapkin J, Abrams D I, Silverberg M J, MacArthur R D, et al. Terry Beirn Community Programs for Clinical Research on AIDS (CPCRA). CD4 count and risk of non-AIDS diseases following initial treatment for HIV infection. AIDS 2008; 22: 841-848.

48.- Prosperi MC, Cozzi-Lepri A, Castagna A, Mussini C, Murri R, Giacometti A, et al. Incidence of malignancies in HIV-infected patients and prognostic role of current CD4 cell count: evidence from a large Italian cohort study. Clin Infect Dis 2010; 50: 1316-21.

49.- El Sadr W M, Lundgren J D, Neaton J D, et al. CD4+ count-guided interruption of antiretroviral treatment. N Engl J Med 2006; 355: 2283-96.

50.- Powles T, Robinson D, Stebbing J, Shamash J, Nelson M, Gazzard B, et al. Highly active antiretroviral therapy and the incidence of non-AIDS-defining cancers in people with HIV infection. J Clin Oncol 2009; 27: 884-90. 